\title{
The maintenance and management system of Zhoushan Trans-sea Bridge
}

\author{
Lianying Zhou', Houbin Fan ${ }^{2}$, Juan Liao ${ }^{3}$, Chao Yang ${ }^{4}$ and Hao Tian $^{5}$ \\ 1 Zhejiang University City College, Hangzhou 310015, China \\ ${ }^{2}$ Zhejiang Zhoushan Cross-Sea Bridge Co., Ltd., Zhoushan 316031, China \\ ${ }^{3}$ Zhejiang University City College, Hangzhou 310015, China \\ ${ }^{4}$ Zhejiang Institution of Communications Hangzhou 311112, China \\ ${ }^{5}$ Zhejiang Scientific Research Institute of Transport, Hangzhou 310006
}

\begin{abstract}
The maintenance management system with respect to Zhoushan Trans-sea Bridge is detailed introduced in this paper. The management system is mainly made up of two parts: Structure Health Monitoring System and Electronic Manual Inspection System. For Structure Health Monitoring System, the components, the service condition, and the evaluation are detailedly discussed. For Electronic Manual Inspection System, the main contents of the inspection management manual and the components of ScanPrint system are briefly introduced.
\end{abstract}

\section{Introduction}

Zhoushan Trans-sea Bridge, composed of Jingtang Bridge, Xihoumen Bridge, Taoyaomen Bridge, Xiangjiaomen Bridge, Cengang Bridge and the junctions, is a key component of Yong-Zhou highway in national highway net. Xihoumen Bridge is a two-span continuous steel box-girder suspension bridge and its main span is $1650 \mathrm{~m}$, which is the second longest and the longest suspension bridge in the world and in China, respectively. It is also the worldwide longest split steel box-girder suspension bridge. Jingtang Bridge is the longest bridge in the five bridges of the Zhoushan Trans-sea Bridge. The main navigation channelis a five-span steel girder cablestayed bridge with a $620 \mathrm{~m}$ main span.

In order to capture the real-time structural state and prevent the disastrous accidents, a structural management system with the advanced technology and the appropriate measures for bridge operating environment is established based on the modern transducer technology, the signal analysis, the long-range intelligent control, and so on ${ }^{[1-4]}$. The integrated management system mainly consists of the Electronic Manual Inspection System (EMIS).

\section{The system configuration}

The EMIS established in Zhoushan Trans-sea Bridge is mainly composed of two parts: the inspection \& maintenance manual and the ScanPrint system. As a programmatic document of the structural inspection \& maintenance system, the inspection \& maintenance manual includes the definition and description of all the contents included in the inspection and maintenance ${ }^{[5-7]}$. The ScanPrint system is an electronic version of the inspection \& maintenance manual, which is mainly used as the record and management of structural damage information, as well as the analysis of the structural operation state and service level.

\section{Application of the EMIS}

\section{1 the Inspection \& Maintenance Manual}

In order to solve the practical problems involved in the maintenance and management of Xihoumen Bridge and Jintang Bridge, the guidance documents with respect to the inspection and maintenance of the two bridges, that are Xihoumen Bridge Inspection \& Maintenance Manual and Jintang Bridge Inspection \& Maintenance Manual, are drawn up according to the individual characteristics of Xihoumen Bridge and Jintang Bridge, based on the review of the maintenance and management of the existing long-span bridges, the reference of the experience, and the most advanced ideas and methods relating to the bridge maintenance and management ${ }^{[5]}$.

\subsubsection{Overview of Inspection and Maintenance}

The basic objective of inspection and maintenance is: (1) to guarantee the bridge structure is not out of service due to the functional deterioration; (2) to reach the allowable maintenance standard with the minimum cost;

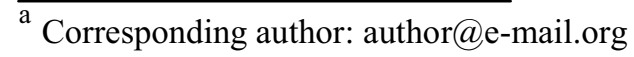


and (3) to keep the bridge structure with the upstanding operation state during the design service life. Therefore, the risk and quantitative management is introduced, the unit level-based bridge management is achieved, and a custom-made preventative inspection and maintenance project with respect to Xihoumen Bridge and Jintang Bridge is provided based on the structural risk assessment. The inspection plan, in which the proportional effect, the minimum cost, and the high efficiency are considered, can provide the substantial basis relating to the inspection management. Regarding the positions and members easy to abrasion and (or) degradation, the inspection frequency should be relatively high, the repair and replacement should be the part of the routine maintenance, and the corresponding reserve items should be put on the maintenance plan. During the maintenance, the damaged and (or) deteriorated members should be substituted by the reserve item using the least time in order to minimize the time of traffic disruption. The pertinent working procedure, which is composed of the maintenance method, the explanation and experimental condition of relative material, the technological standard, the inspection level and range, the testing standard, and the standard and requirement of inspection record including material supply, should be drawn up with respect to each daily or particular maintenance method. The inspection and maintenance procedure is shown in Figure 1.

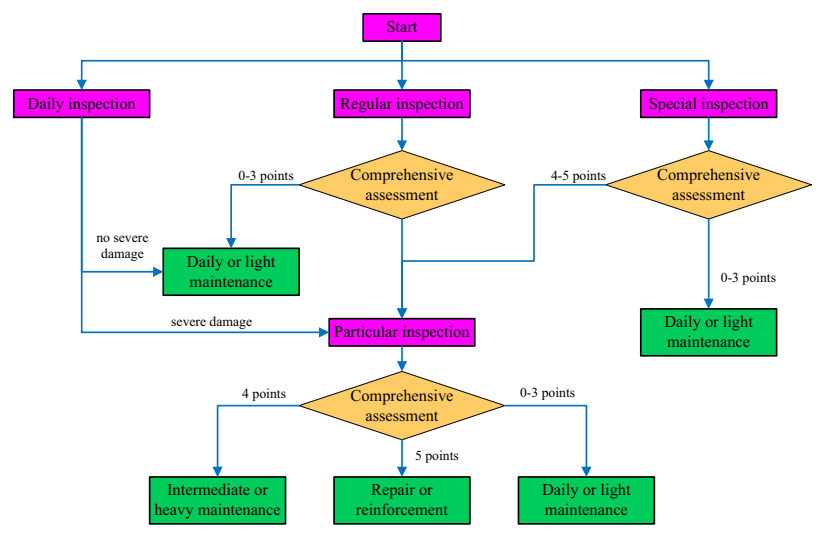

Figure 1 Inspection and maintenance procedure

\subsubsection{Classification of Inspection}

According to the effect and the time period, the inspection is classified as five types: Initial Inspection, Daily Inspection, Regular Inspection, Special Inspection and Particular Inspection.

(1)Initial Inspection. Initial Inspection is a comprehensive inspection when the structure is just finished and has not gone into service, or the structure is just reinforced or rebuilt.

(2)Daily Inspection. Daily Inspection is a routine inspection with respect to the technical condition of deck subsidiary facilities, superstructures, substructures, as well as accessory structures.

(3)Regular Inspection. Regular Inspection is a risk assessment-based periodical inspection. This inspection covers all structure and its accessory structures. It aims at evaluating the structural serviceability and providing the basic data relating to the maintenance and management project. According to the actual characteristics of various structural members, Regular Inspection is divided into three levels and the corresponding inspection frequencies are:

A-grade Inspection: semi-annual inspection.

B-grade Inspection: annual inspection.

C-grade Inspection: triennial inspection.

(4)Special Inspection. Special Inspection is an emergency-reacting inspection, which is triggered when the disasters such as typhoon, earthquake, ship collision, car accident, and fire are occurred.

(5)Particular Inspection. Particular inspection is a pertinent inspection based on the abovementioned Daily Inspection, Regular Inspection and Special Inspection. It aims at further evaluating the damage degree, analyzing the damage cause, and predicting the damage development, which is composed of onsite inspection, indoor test and calculation analysis, etc.

\section{2 the ScanPrint System}

The ScanPrint system, developed by the French Advitam company, is introduced in the EMIS. This product has been successfully employed in the maintenance and management systems established in many long-span bridges. The chinesization is performed and the Chinese codes are introduced in ScanPrint system by the bridge administration. The Electronic Manual Inspection System is thus established, based on the database and all-electronic input. The framework of ScanPrint system is shown in Figure 2.

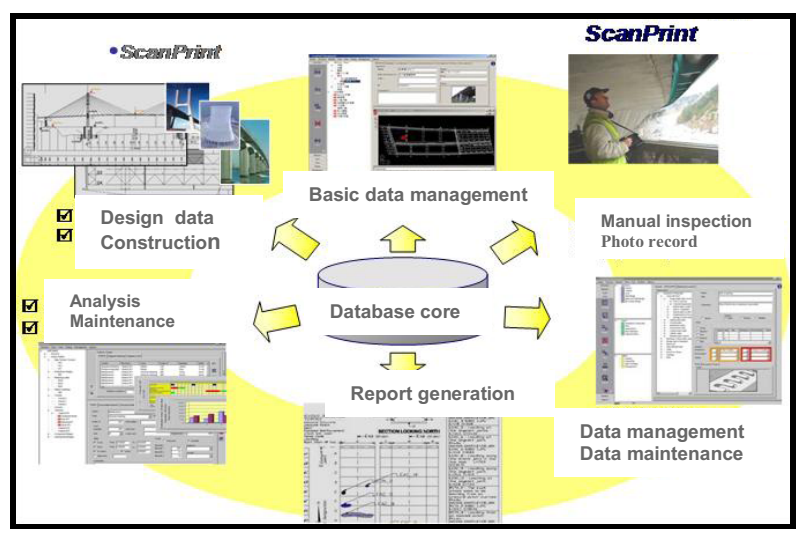

Figure 2 The framework of the ScanPrint system

The ScanPrint system is composed of four sections: the Database management module, the Inspection management module, the Onsite inspection module, and the Analysis report module.

\subsubsection{Database Management Module}

The Database management module is the management core of ScanPrint, and its main function includes: the structure information management, the database management, as well as the prediction and analysis of maintenance cost. Furthermore, the structure information management consists of the engineering 
information (such as the design, the construction, the location, the photos, and the drawings), the inspection forms, and the damage grade criterion.

\subsubsection{Inspection Management Module}

The main function of the Inspection management module is: (1) defining the inspection program, choosing the structure and members should be included in the inspection program, and filling out the inspection drawings, inspection forms as well as the other relating information; (2) exporting the sub database of the inspection program, which is installed in the portable computer on the site; (3) merging the inspection program, in which the sub database including the damage and grade information is merged into the master database when the inspection program is finished by using the onsite computer; and (4) inputting the damage photos defined in the inspection sub database.

\subsubsection{Onsite Inspection Module}

The main function of the Onsite inspection module is: (1) finishing the on-site inspection program; (2) recording the damage on the built-in AutoCAD-based inspection drawings; (3) describing the damage according to built-in damage database; (4) supplementally describing the damage by means of drawing sketch and adding notes; (5) replying the questions of the different inspection locations on the inspection forms; (6) grading the damage and the members; (7) defining the damage photos; and (8) reminding the engineers whether the inspection program is finished or not by using different colors in case of missing any locations.

\subsubsection{Analysis Report Module}

The main function of the Analysis report module is: (1) generating Word- or Excel-based inspection reports; and (2) comparative analysis and predicting for damage.

\section{Conclusions}

Regarding as the ten-billion project of Zhejiang Province and the national star project, the maintenance and management during the service life is extremely difficult with respect to Zhoushan Trans-sea Bridge, which is composed of many bridge types such as the long-span suspension bridge, the cable-stayed bridge, the continuous rigid-frame bridge, and the continuous girder bridge, considering not only the technology requirement but also the social concern[7,8]. Based on more than two years on-site management, a set of relatively comprehensive bridge maintenance management system is primarily established by the bridge administration, in which the Electronic manual inspection system are combined to basically achieve the real-time monitoring towards the key structural responses and the surrounding environmental conditions of the entire trans-sea bridge. However, many technical problems involved in the specific work of the maintenance and management are still existed. As a result, these problems are urgently needed to be solved by the bridge administration and the existing maintenance and management system can thus be gradually improved.

\section{Acknowledgements}

This study was supported by the Department of Transportation and Communications of Zhejiang Province through the Scientific and Technological Project (Grant No. 2012H06), the Department of Science and Technology of Zhejiang Province through the Qianjiang Talents Project (Grant No. 2012R10082), Zhejiang Communications Investment Group CO., LTD through the Scientific and Technological Project (Grant No. 201101), and the Department of Transportation and Communications of Zhejiang Province through the Scientific and Technological Project (Grant No. 2013H25). The support is gratefully acknowledged.

\section{References}

1. Xu You-lin, Chen Zhi-wei Mult iloading Long Span Suspension Bridges with St ructural Health Monitoring Systems. In: Journal of Disaster Prevention and Mitigat ion Engineering , 30(9): 394 399(2010)

2. Liu T T , Xu Y L, Zhang W S, etal. Buffet ingInduced str esses in a long suspension bridge: Fat igue and Reliability: structural healt $\mathrm{h}$ monitoring or ientated stress a nalysis[ J] . Wind and Structures(2009).

3. Xu Y L, Chen Z W. Advances in wind- vehicle-lng span br idge inter action [ C] // Proceedings of the Fourth Inter nationa 1 Symposium on Envir onmental Vibrat ion: Prediction, Monitoring, and Evaluation,Beijing: [ s. n. ] (2009)

4. Peng D p, Jin Y Y, etal. Study on the structural health and safety monitoring system of Hangzhou Bay Cross Sea Bridge[ J]. Highway (2015)

5. CCCC Highway Consultants CO., LTD. Training manual of the hardware in structure health monitoring and management system of Xihoumen Bridge and Jintang Bridge in Zhoushan islandmainland link Project(2009)

6. CCCC Highway Consultants CO., LTD. Training manual of the software in structure health monitoring and management system of Xihoumen Bridge and Jintang Bridge in Zhoushan island-mainland link Project( 2009)

7. CCRISOS Co., LTD. Inspection and maintenance manual of Xihoumen Bridge in Zhoushan islandmainland link Project( 2011) 